\title{
Free Flap Reconstruction of the Head and Neck Region: A Series of 127 Flaps Performed by Otolaryngologists
}

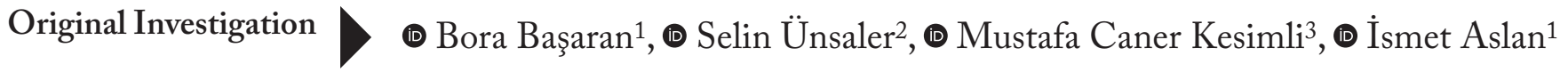 \\ ${ }^{1}$ Department of Otolaryngology Head and Neck Surgery, İstanbul University-İstanbul Faculty of Medicine, İstanbul, \\ Turkey \\ ${ }^{2}$ Department of Otolaryngology Head and Neck Surgery, Koç University School of Medicine, İstanbul, Turkey \\ ${ }^{3}$ Department of Otolaryngology Head and Neck Surgery, İstinye University Faculty of Medicine, İstanbul, Turkey
}

\section{Abstract}

\section{ORCID ID of the authors: \\ B.B. 0000-0003-0546-2848; S. Ü. 0000-0001-7108-9194; M.C.K. 0000-0003-1675-0394; \\ I.A. 0000-0001-6994-9490.}

Cite this article as: Başaran B, Ünsaler S, Kesimli MC, Aslan I. Free Flap Reconstruction of the Head and Neck Region: A Series of 127 Flaps Performed by Otolaryngologists.. Turk Arch Otorhinolaryngol 2021; 59(2): 103-10.

\section{Corresponding Author:}

Bora Başaran; bbasaran@istanbul.edu.tr

Received Date: 26.01.2021 Accepted Date: 18.04 .2021

Content of this journal is licensed under a Creative Commons Attribution 4.0 International License. Available online at www.turkarchotolaryngol.net
Objective: To determine flap success rate and complications in patients who underwent microvascular free tissue reconstruction after major head and neck ablative surgery and to report the improvement in the results.

Methods: Medical records of 124 patients who were operated on in 2012 to 2019 were retrospectively reviewed. Indications for different free flap types, success rates and re-exploration rates, donor site morbidities, and reasons for flap loss were analyzed. Patients were divided into two groups to identify the effects of the anticoagulant and the antiaggregant treatments on postoperative results.

Results: There were 127 flaps in 124 patients, including two different free flaps each in three patients that were harvested and used in the same surgical session. Of the total 127 flaps, 82 (64.6\%) were radial forearm flaps, 39 (30.7\%) were fibula flaps, and 6 (4.7) were rectus abdominis flaps. Four patients were re-explored for flap perfusion problems, and 18 patients were re-explored for hematoma drainage $(n=22 / 124, \% 17.3)$. The rate of hematoma and re-exploration was higher in patients who received anticoagulant and antiaggregant treatments synchronously $(p=0.02)$. Three flaps were lost, and the overall success rate was $97.6 \%$. Two patients died from perioperative complications. No major complications were observed at the donor site; minor complications were observed in 30 patients.

Conclusion: The success rate for the 127 flaps in 124 patients were found comparable to those reported in the literature. These results show that successful outcomes can be achieved with experience and a head and neck team dedicated to improving the results in microsurgical reconstruction, and flap failure rarely occurs if perioperative care of the patients is given meticulously.

Keywords: Head and neck cancer, reconstructive surgery, microsurgical free flaps, otolaryngologists 


\section{Introduction}

Survival from head and neck cancers continues to improve with the help of early diagnosis and the advancement in treatment strategies (1). As a principle, the primary goal of the cancer treatment is to achieve good oncological control. This is followed by the goal of rehabilitating functional loss and cosmetic defects with utmost care. Cancer survivors can maintain their social and economic position as long as their quality of life is not significantly affected. Whilst being the anatomic region of the sense organs and the most important part of the body in a person's social interaction, the head and neck is also the region where significant functional and cosmetic loss occurs as a result of cancer treatment $(2,3)$.

There are local flaps, rotational flaps, and pedicle flaps described to be used for the reconstruction of the surgical defects after primary ablation or salvage surgery of the head and neck tumors. Free flaps, on the other hand, are considered as the gold standard in head and neck reconstruction surgeries since they supply multiple different tissues, such as skin, muscle, bone, and nerve, without limitation of transfer to the surgical resection site $(4,5)$. As a result, free flaps provide better cosmetic and functional outcomes. They also offer the advantage of allowing two surgical teams to work at the same time. Additionally, harvesting a tissue distant from the head and neck region can be advantageous in patients previously treated for head and neck cancer. In this study, the authors report their experience in free flap reconstruction, as well as their success rates, complications and perioperative morbidities.

\section{Methods}

In this study, the medical records of 124 patients who underwent free flap reconstruction by otolaryngologists following a major head and neck surgery in the period September 2012 through December 2019 were retrospectively reviewed. Of the 124 patients, 57 were female (45.9\%), 67 were male (54.1\%); their mean age was 45.9 (range: 14-72). All patients were evaluated by the tumor board consisting of head and neck surgeons, radiation oncologists, medical oncologists, a nuclear medicine physician, a radiologist, and a pathologist. The patients were informed about the possible surgical complications and postoperative morbidities, and informed consent was preoperatively obtained from all participating patients. The study was approved by the Institutional Ethics Committee of İstinye University Faculty of Medicine (decision no: 2/2021.K-02).

Ablative surgery and reconstruction were performed in the same surgical session in all patients. Three different types of free flaps were used: fasciocutaneous radial forearm flap (RFF), osseocutaneous fibula flap (FF), and musculocutaneous rectus abdominis flap (RAF). RFF and FF flaps were harvested under tourniquet from the extremities, and loop magnification (x2.5 or x3.5) was used in all. The Allen test, by which the patency of the ulnar artery is assessed, was done in all RFF patients; computerized tomography (CT) angiography of the lower extremities was done in all FF patients to exclude the presence of arteria peronea magna anomaly or atherosclerotic plaque.

The facial artery, lingual artery or superior thyroid artery were used for arterial anastomosis. Venous anastomosis was done to the thyroid vein or the thyrolingofacial trunk, or directly to the internal jugular vein as end-to-side anastomosis. Anastomoses were done under microscopic magnification with $9-0$ or $10-0$ polyamide suture with round needle. All patients were followed in the intensive care unit for one day to keep the blood pressure stabilized, and then transferred to the ward the next day. All patients were given anticoagulant treatment with enoxaparin sodium and some patients were also given antiaggregant treatment with acetylsalicylic acid.

The indications for the preference of free flaps, the success rate of different flap types, and the risk factors for flap loss were analyzed. The need for revision surgery, the morbidities of the donor site, and the postoperative complications that developed on the resection site were reviewed. Patient data were also analyzed by dividing the patients into two groups, as patients operated on in September 2012-August 2016 (group 1) and those operated on in September 2016December 2019 (group 2), to identify the effects of the anticoagulant treatment on the postoperative results.

The statistical analyses were performed with the Statistical Package for the Social Sciences for Mac version 21.0 (IBM SPSS Inc.; Armonk, New York, USA). Fischer's exact test was used in analyses and $\mathrm{p}<0.05$ was considered significant.

\section{Results}

Free flap reconstruction was performed during salvage surgery in only five patients (4\%), of whom three had sinonasal malignancy (surgery+, RT+), and two had tongue cancer (surgery+, RT-). RAF was preferred to preserve the dura mater and to close the defect which resulted from craniofacial resection in two patients, and to close the total maxillectomy defect in one patient; RFF was used for the reconstruction of the tongue after subtotal glossectomy. Free flaps were preferred in the primary surgical treatment of the remaining 119 patients. The final pathological diagnosis of the patients are given in Table 1.

In total 127 flaps were used in 124 patients as three patients received synchronous two free flaps in the same surgical session. Of the 127 free flaps, 82 were RFF (64.6\%), 39 were FF (30.7\%), 6 were RAF (4.7\%). The defects in the surgical resection sites and the flaps used for their reconstruction are listed in Table 2. In three patients with oral cavity cancer, reconstruction was achieved by double free flaps as FF and 
RFF, which were designed for mandibular reconstruction in all three patients: reconstruction of the tongue in two patients and closure of the skin defect over mentum in one patient.

Recipient veins, donor arteries and type of the anastomoses were analyzed (Table 3). Except for one patient all anastomoses were done to the vessels on the side in which the flap was predominantly set. In one patient, RFF was anastomosed to the vessels on the contralateral side and then lost due to arterial insufficiency.

Anticoagulants and antiaggregants were routinely administered to all patients in group $1(n=63)$ with the following doses: enoxaparin sodium 2x4000 IU, acetylsalicylic acid 1 x100 mg. Because of the high rates of postoperative hematoma, antiaggregant treatment was abandoned in patients in group $2(\mathrm{n}=61)$ unless the patient had another disease that necessitated antiaggregants. Additionally, the dosage of enoxaparin was reduced to 6000 IU.

\section{Complications}

Four patients underwent surgical re-exploration because of flap perfusion problems (3.2\%). In two FFs, the problem was venous insufficiency that developed in the postoperative 24 hours in both. In two RFFs, the problem was arterial insufficiency, which developed in the postoperative 24

Table 1. Final pathological diagnosis of patients

\begin{tabular}{|l|l|l|}
\hline Histological type & Number of patients & Percentage (\%) \\
\hline Squamous cell carcinoma & 100 & $80.6 \%$ \\
\hline Osteosarcoma & 7 & $5.6 \%$ \\
\hline Ameloblastoma & 5 & $4 \%$ \\
\hline Adenoid cystic carcinoma & 2 & $1.6 \%$ \\
\hline Mucoepidermoid carcinoma & 2 & $1.6 \%$ \\
\hline Other* & 8 & $6.5 \%$
\end{tabular}

*Adenosquamous carcinoma $(n=1)$, basal cell adenocarcinoma $(n=1)$, giant cell bone tumor $(n=1)$, esthesioneuroblastoma ( $n=1)$, Ewing sarcoma ( $=1)$, hemangioma $(n=1)$, ossifying fibroma $(n=1)$ and aseptic necrosis of mandibula due to biphosphate usage $(n=1)$

n: Number

Table 2. Defects in the surgical resection sites, flaps used for their reconstruction, and failed flaps according to reconstruction site

\begin{tabular}{|l|l|l|}
\hline & RFF & FF \\
\hline Partial pharyngectomy & 12 & \\
\hline Total laryngopharyngectomy & 9 & \\
\hline Buccal region (mucosa or through and through defect) & 12 & \\
\hline Total lower lip & 1 & 1 \\
\hline Tongue and oral base & $47(2)^{*}$ & \\
\hline Skin of submental and submandibular region & 1 & $35(1)^{* *}$ \\
\hline Mandible & & 4 \\
\hline Maxilla & & 3 \\
\hline Craniofacial resection & & 2 \\
\hline
\end{tabular}

RFF: Fasciacutaneous radial forearm flap, FF: Osseocutaneous fibula flap, RAF: Musculocutaneous rectus abdominis flap

*Two flaps (RFF) used for tongue defect reconstruction failed

**One FF used for mandibulectomy defect failed

Table 3. Vascular anastomoses of flaps

Arterial anastomoses

Facial artery $(\mathrm{n}=111 / 87.4 \%)$

Superior thyroid artery $(\mathrm{n}=12 / 9.7 \%)$

Lingual artery $(\mathrm{n}=2 / 1.6 \%)$

External carotid artery - end-to-end $(n=2 / 1.6 \%)$

Internal jugular vein - end-to-side $(n=66 / 53.2 \%)$

Thyrolingofacial trunk $(\mathrm{n}=59 / 47.6 \%)$

Middle thyroid vein $(n=2 / 1.6 \%)$ 
hours in one patient and on the postoperative $14^{\text {th }}$ day in another patient because of a salivary fistula to the neck. The anastomoses were explored, sutures were untied and washed with heparin and lidocaine solution. Only one flap (FF) survived; the other three flaps were lost (75\%). These four patients were all in group 1, i.e., they were receiving highdose anticoagulant and antiaggregant treatment. Including these four patients, 22 patients in total underwent surgical re-exploration (17.7\%). The reason for re-exploration was hematoma in the neck in 18 patients, so the rate of hematoma was found to be $14.5 \%$. Of these 18 patients, 12 were in group $1(12 / 63, \% 19)$, and six were in group 2 (6/61, \%9.8). The difference between these rates were found statistically significant $(\mathrm{p}=0.02)$.

Overall, only three flaps (1 FF, 2 RFFs) were lost among the 127 flaps; the success rate was found to be $97.6 \%$. While these three losses were in group 1 ( 3 out of 65 ), there was no flap loss among the 62 flaps in group 2. The difference between these two groups regarding flap loss was not statistically significant $(\mathrm{p}=0.2445)$.

\section{Morbidity and mortality}

The donor site morbidities were analyzed; there was no healing problem on the donor site in 97 flaps (97/127; 76.4\%). The donor site was repaired with split-thickness skin graft in all patients with RFF; partial graft necrosis occurred in 14 patients (\%17.1) and defects closed by secondary intention in all 14 patients in two months after debridement. No motor or neurologic sequel of the forearm occurred in any of the patients. There was hematoma of the donor site in one patient and keloid, which required steroid injection, in another patient. In three of the FF cases, contracture of the toes with a configuration of dorsiflexion developed, but rehabilitation was achieved without sequel in two patients and with a partial sequel in one patient. Of the seven patients in whom the donor site was repaired primarily, three had necrosis of the skin in the peroneal region. In one of these patients the wound was left to healing by secondary intention after debridement. In the remaining two the findings in the crus were considered to be risky for compartment syndrome, because their legs were thick and fatty, so vacuum assisted closure was applied. Of the 32 patients whose peroneal skin were repaired with split thickness skin graft, nine had partial necrosis of the graft (28\%) and the defect closed by secondary intention in two months after debridement in all. The defects were repaired primarily in the donor site of RAF; in one patient hematoma occurred in the first 72 hours and was controlled after changing the drain tube, and no abdominal hernia developed in any of the patients. While the overall average hospitalization time of the patients was 14.2 days, according to flap types, 13.4 days for RFF; 16.6 days for FF; and 10.3 days for RAF.
Two patients (1.6\%) died due to perioperative complications. One of them had undergone craniofacial resection including resection of the dura and the brain parenchyma because of recurrence of esthesioneuroblastoma and the defect was reconstructed with RAF. She developed subdural hematoma on the $5^{\text {th }}$ postoperative day. Despite craniotomy and drainage, she died on the $15^{\text {th }}$ postoperative day because of parenchymal edema and herniation. The other patient had undergone subtotal glossectomy and reconstruction with RFF; she died due to a rupture of the innominate artery caused by a tracheostomy complication on the $14^{\text {th }}$ postoperative day. There was no flap viability problem in these two patients.

\section{Discussion}

The first free flap reconstruction performed by otolaryngologists was reported by Panje et al. (6) from Iowa in 1976 for the reconstruction of the oral cavity. Today, microvascular surgery training has become a part of the head and neck surgery fellowship programs in most of the centers in Western countries, and head and neck surgeons perform microvascular reconstruction themselves $(7,8)$. This, however, is not the situation in Turkey, and there is not yet a fellowship program in otolaryngology. The otolaryngology residents cannot train in microvascular surgery during their 5 -year residency program. Therefore, reconstruction of the head and neck region by otolaryngologists is being performed only in a few centers. In other centers, plastic surgeons take over the reconstruction stage of the surgery, or if this is not possible, local/pedicled flaps are preferred, which may cause a significant decrease in the quality of life of the patient in the rest of his/her life.

On the other hand, the duration of free flap operations is long, and they require two surgical teams working together for the ablation of the tumor and for the harvesting of the flap at the same time. Additionally, flap harvesting requires anatomical knowledge of a region different from the head and neck. These operations necessitate postoperative admission of the patients in the intensive care unit at least for one night, and require longer hospitalization times and higher healthcare costs (9). Whilst any kind of a defect in the head and neck region can be repaired by free tissue transfer, free flaps are not the first choice of the authors because of the mentioned reasons, and they opt for this type of flaps in cases of absolute indication. Reconstruction should be planned individually for every patient based on the three-dimensional anatomical features of the defect. If possible and sufficient, local flaps or pedicled flaps should be preferred since they are easier to perform and cause less morbidity in the early postoperative period.

There are numerous alternatives to free tissue flaps in the human body when perforator flaps are also included, however, 
the most commonly used flaps in head and neck region are RFF, FF, RAF, anterolateral thigh, jejunum, iliac crest, and scapular free flaps $(8,10)$. It is assumed that the variety of flaps will increase as the experience of the microvascular surgeon increases. If, however, we look from the perspective of an otolaryngologist who is interested in head and neck reconstruction, the aim of the reconstruction should be to achieve the best functional result for the patient with minimal morbidity. The flaps described in this study were suitable and allowed the two surgical teams work together simultaneously. The other alternatives were not preferred because of different disadvantages such as: anterolateral thigh flap has a high rate of anatomical variations (11); jejunum flap requires intraabdominal intervention and participation of a general surgeon; harvesting the iliac crest flap has the risk of penetration into the abdomen; harvesting of the scapular flap requires prone positioning of the patient, and thereby does not allow for the simultaneous work of two teams. As demonstrated in our study, RF and FF meet the $90 \%$ of the free flap requirement for head and neck reconstruction, and together with the RAF, these three types of flaps fulfill the need. RFF is preferred when there is a need for fine tissue; the fascia and the skin are sufficient for the repair of the tongue (Figure 1a), the pharynx and the hypopharynx. FF is preferred when bone tissue is needed for the reconstruction of the maxilla and the mandible (Figure 1b). RAF is preferred when there is a need for bulky tissue for the restoration of the defects after total maxillectomy (Figure 1c) and total glossectomy.

FF, iliac crest, radial forearm osseocutaneous flaps and scapular flaps can be used for the reconstruction of the upper and lower jaws; in this series, FF was preferred because of the surgeons' experience. In patients with oral cavity cancer and accompanying infiltration of the skin, free tissue transfer may be indicated for not only the repair of the oral cavity structures but also for repairing the skin. With this indication,

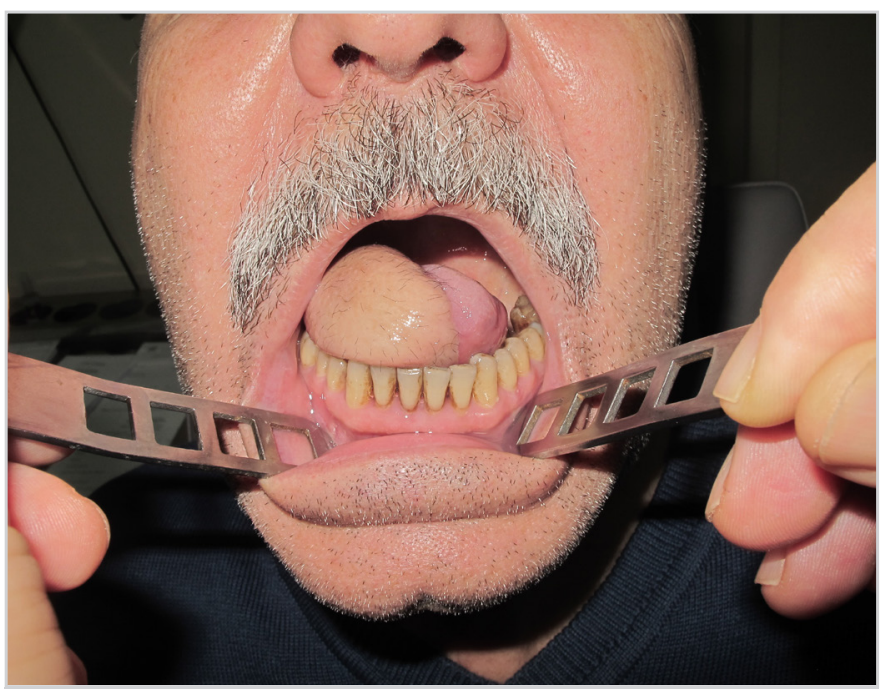

Figure 1a. Reconstruction of the tongue with radial forearm flap
$\mathrm{RFF}$ and FF were performed in the same surgical session in one patient with mandibular involvement, and RFF was used for another patient without bone involvement. In cases of large skull base defects resulting from craniofacial resection, free flap reconstruction provides the tissue to restore the defect and separate the intracranial content from the nasal cavity (12). Therefore, RFF and RAF can be preferred for this purpose, and RAF was used in our series. In patients with advanced-stage hypopharynx tumors that require total pharyngectomy and end up with circular pharyngeal defect, the pharynx should be reconstructed by folding the flap tissue to form a tube as a neopharynx. An anterolateral thigh flap or RFF can be used for this purpose, while the jejunum flap is also an alternative. In our series, RFF was

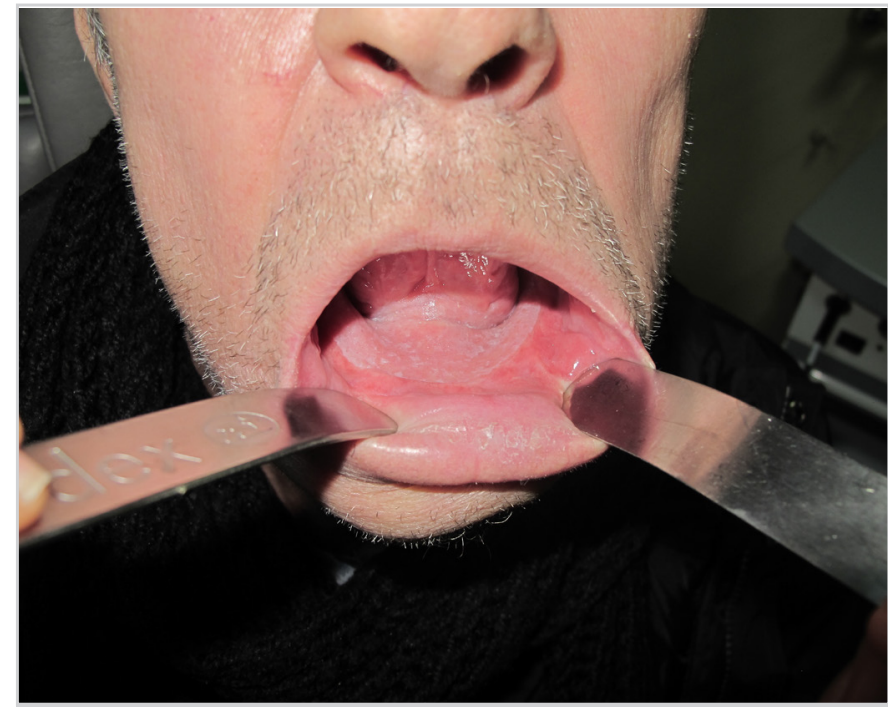

Figure 1b. Reconstruction of the mandible and floor of mouth with fibula flap

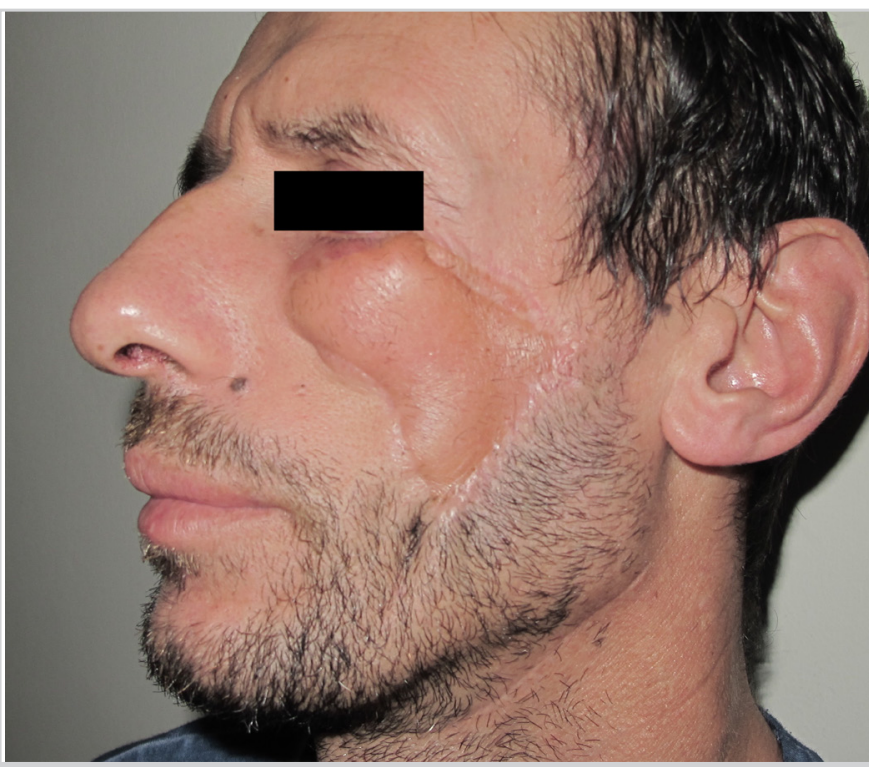

Figure 1c. Restoration of maxillectomy defect and skin defect with rectus abdominis flap 
preferred, and we observed no stricture at the esophageal anastomosis line in any of the patients, and rehabilitation of swallowing was achieved in all. RFF can also be used for partial pharyngectomy defects, yet pedicled flaps are also suitable for this purpose. The fine structure of RFF, which enables it to be shaped easily, and better postoperative functional results are the reasons why it is preferred for the reconstruction of the partial pharyngeal defects (13).

In the postoperative period, a free flap surgeon should be prepared to deal with surgical field complications not only in the head and neck region but also in the donor site. No major complications developed in any patient in our series. In the literature, the most serious complication following RFF harvesting is insufficient arterial supply of the hand by ulnar artery (14). The deep palmar arch of the hand is mainly formed by the radial artery with the contribution of the ulnar artery, which mainly forms the superficial palmar arch. The anastomosis between them is tested by the Allen test (15). In a cadaver study, it was demonstrated that arterial supply of the hand by ulnar artery was insufficient in $12 \%$, however, this study dates to 1961, and further studies are needed (14). The Allen test was performed in all 82 patients in our series and no insufficiency was observed in the test and neither did any postoperative ischemia occur.

$\mathrm{FF}$ is riskier for donor site complications. The arterial supply of the crus is from the popliteal artery which divides into two as anterior tibial artery, and the posterior tibial artery which gives rise to the peroneal artery that supplies the FF. In $5 \%$ of the general population, anterior and posterior tibial arteries can be hypoplastic, which leaves the arterial supply of the crus to be solely from the peroneal artery, and this variation of the arterial system is called peronea magna (16). Therefore, the arterial system of the lower extremities of patients who are planned to undergo FF harvesting should be evaluated radiologically to exclude the presence of peronea magna variation $(17,18)$; for this purpose, doppler ultrasonography, CT angiography, magnetic resonance angiography or conventional angiography can be used. Another benefit of radiological studies is the ability to recognize an occlusive atherosclerotic disease of the femoral arterial system. Atherosclerosis is more frequent in the lower extremities, and if we consider that a high percentage of head and neck cancer patients are smokers, we may estimate this risk to be higher in them (19). In our series, all patients were evaluated by CT angiography preoperatively; two patients were assessed to have peronea magna so cardiovascular surgeons performed peroneal artery bypass surgery, which was successful in both patients. Additionally, the planning of closure of the peroneal region should be done with care in patients with fatty crus. Harvesting of a RA flap may lead to the development of an abdominal hernia. During the closure of the donor site, the use of a mesh decreases the incidence of hernia formation (15). We did so in each RAF case, and no hernia was observed in the long-term postoperative follow-up.

Although free flap surgery has specific difficulties in the different stages of the procedure, including harvesting of the flap and flap inset, the most important stage that determines the success of the surgery is vascular anastomosis. The problems in anastomosis usually develop in the first 24 hours; the endothelization of the tunica intima is completed in the first 72 hours so complications related to flap vascularization rarely occur after 72 hours (20-22). The use of antiaggregants and anticoagulants to prevent the development of thrombosis until the completion of the endothelialization of the intima is usually considered useful, but is still controversial. In the literature, while some authors recommend their routine usage, some others recommend it only in patients with high risk of atherosclerosis or in those with previous flap failure (23-25). The most frequently used agents are acetylsalicylic acid, enoxaparin and dextran 40; however, none of them are proved to be clinically efficient in free flap survival (26-29). One fifth of the patients in group 1 were explored in the operating room because of development of a hematoma. This result led the authors to change the treatment regimen, after which the rate of hematoma decreased by half, and no flap loss occurred. However, it is not possible to speculate on the effects of anticoagulant and antiaggregant treatments on the flap success rate, because this was not a randomized study. Furthermore, the patients in group 1 were operated on at a time when the surgeons were relatively less experienced. Therefore, it can be recommended to surgeons who are at the beginning of their learning curve to use both anticoagulants and antiaggregants to take the highest precaution against flap failure.Large series in the literature report re-exploration rates of $8 \%-9 \%$ and flap success rates of $95 \%-100 \%(15,22,30)$. In our series flap success rate was $97.6 \%$. At the beginning, the re-exploration rate was $17.7 \%$ and was decreased to $9.8 \%$ in the later period, which is comparable to the rates reported in the literature. The fact that there was no flap failure in group 2 can be explained by the authors' increased surgical experience. Although this decrease is attributed to the modification of the anticoagulation protocol, the effect of the increased experience of the surgeons cannot be denied.

\section{Conclusion}

Free flaps widen the limits of intervention in ablative surgery. Factors such as the requirement of education of microvascular surgery, long operating times, and detailed postoperative care of patients discourage the head and neck surgeon from performing microvascular reconstruction. As demonstrated in this study, however, free tissue transfer can be performed by otolaryngologist with the same success rates as reported in the literature, when the head and neck team is dedicated to achieving the best reconstruction methods. The 
proficiency of the surgeon increases rapidly when the correct surgical steps are followed, and flap failure rarely occurs if perioperative care is given meticulously according to the recommendations.

\section{Main Points}

- Head and neck reconstruction following cancer surgery may be performed by otolaryngologists successfully with the same success rates as reported in the literature.

- Head and neck surgeons who plan to start with free flap surgery could concentrate on fasciocutaneous radial forearm flap and osseocutaneous fibula flap, which meet $90 \%$ of the free flap requirement for head and neck reconstruction.

- It is recommended to surgeons who are at the beginning of their learning curve with microvascular surgery to use both anticoagulant and antiaggregant therapies in order to take the highest precaution against flap failure.

- With the increase in surgical experience, anticoagulant and antiaggregant therapies may be reduced, unless the patients had another disease requiring continuous antiaggregant usage. This may reduce the risk of postoperative hematoma and the need for re-exploration.

Ethics Committee Approval: The study was approved by the Institutional Ethics Committee of İstinye University Faculty of Medicine (decision no: 2/2021.K-02).

Informed Consent: The patients were informed about the possible surgical complications and postoperative morbidities, and informed consent was preoperatively obtained from all participating patients.

Peer-review: Externally peer-reviewed.

\section{Authorship Contributions}

Surgical and Medical Practices: B.B, S.Ü., M.C.K., İ.A., Consept: B.B., S.Ü., İ.A., Design: B.B., S.Ü., Data Collection and/or Processing: B.B., S.Ü., M.C.K., İ.A., Analysis and/or Interpretation: B.B., S.Ü., M.C.K., Literature Search: B.B., S.Ü., Writing: B.B., S.Ü., M.C.K., İ.A.

Conflict of Interest: No potential conflict of interest relevant to this article was reported.

Financial Disclosure: The authors declared that this study has received no financial support.

\section{References}

1. Pulte $\mathrm{D}$, Brenner $\mathrm{H}$. Changes in survival in head and neck cancers in the late 20th and early 21 st century: a period analysis. Oncologist 2010; 15: 994-1001. [Crossref]

2. Zimmaro LA, Sephton SE, Siwik CJ, Phillips KM, Rebholz WN, Kraemer HC et al. Depressive symptoms predict head and neck cancer survival: examining plausible behavioral and biological pathways. Cancer 2018; 124: 1053-60. [Crossref]

3. Lydiatt WM, Moran J, Burke WJ. A review of depression in the head and neck cancer patient. Clin Adv Hematol Oncol 2009; 7 : 397-403. [Crossref]

4. Hanasono MM, Matros E, Disa JJ. Important aspects of head and neck reconstruction. Plast Reconstr Surg 2014; 134: 968e-80e. [Crossref]

5. Vila PM, Rich JT, Desai SC. Defining quality in head and neck reconstruction. Otolaryngol Head Neck Surg 2017; 157: 545-7. [Crossref]

6. Panje WR, Bardach J, Krause CJ. Reconstruction of the oral cavity with a free flap. Plast Reconstr Surg 1976; 58: 415-8. [Crossref]

7. Spiegel JH, Polat JK. Microvascular flap reconstruction by otolaryngologists: prevalence, postoperative care, and monitoring techniques. Laryngoscope 2007; 117: 485-90. [Crossref]

8. Kovatch KJ, Hanks JE, Stevens JR, Stucken CL. Current practices in microvascular reconstruction in otolaryngology-head and neck surgery. Laryngoscope 2019; 129: 138-45. [Crossref]

9. Gabrysz-Forget F, Tabet P, Rahal A, Bissada E, Christopoulos A, Ayad T. Free versus pedicled flaps for reconstruction of head and neck cancer defects: a systematic review.J Otolaryngol Head Neck Surg 2019; 48: 13. [Crossref]

10. Disa JJ, Pusic AL, Hidalgo DH, Cordeiro PG. Simplifying microvascular head and neck reconstruction: a rational approach to donor site selection. Ann Plast Surg 2001; 47: 385-9. [Crossref]

11. Rozen WM, Ashton MW, Pan WR, Kiil BJ, McClure VK, Grinsell $\mathrm{D}$, et al. Anatomical variations in the harvest of anterolateral thigh flap perforators: a cadaveric and clinical study. Microsurgery 2009; 29: 16-23. [Crossref]

12. Gil Z, Abergel A, Leider-Trejo L, Khafif A, Margalit N, Amir A, et al. A comprehensive algorithm for anterior skull base reconstruction after oncological resections. Skull Base 2007; 17: 25-37. [Crossref]

13. Başaran B, Ünsaler S. Carcinoma of the posterior wall of the hypopharynx: surgical treatment with larynx preservation. Braz J Otorhinolaryngol 2020: S1808-8694(20)30071-9. [Crossref]

14. Coleman SS, Anson B. Arterial patterns in the hand based upon a study of 650 specimens. Surg Gynecol Obstet 1961; 113: 409-24. [Crossref]

15. Genden EM, Rinaldo A, Suárez C, Wei WI, Bradley PJ, Ferlito A. Complications of free flap transfers for head and neck reconstruction following cancer resection. Oral Oncol 2004; 40: 979-84. [Crossref]

16. Rosson GD, Singh NK. Devascularizing complications of free fibula harvest: peronea arteria magna. J Reconstr Microsurg 2005; 21: 533-8. [Crossref]

17. Futran ND, Stack BC Jr, Zaccardi MJ. Preoperative color flow Doppler imaging for fibula free tissue transfers. Ann Vasc Surg 1998; 12: 445-50. [Crossref] 
18. Manaster BJ, Coleman DA, Bell DA. Magnetic resonance imaging of vascular anatomy before vascularized fibular grafting. J Bone Joint Surg Am 1990; 72: 409-14. [Crossref]

19. Shu J, Santulli G. Update on peripheral artery disease: epidemiology and evidence-based facts. Atherosclerosis 2018; 275: 379-81. [Crossref]

20. Hidalgo DA, Jones CS. The role of emergent exploration in freetissue transfer: a review of 150 consecutive cases. Plast Reconstr Surg 1990; 86: 492-8; discussion 499-501. [Crossref]

21. Schwabegger AH. Flap re-exploration. In: Wei FC, Mardini S, editors. Flaps and Reconstructive Surgery. 1st edition. Saunders Elsevier Publishers; 2009. p.125-36. [Crossref]

22. Bui DT, Cordeiro PG, Hu QY, Disa JJ, Pusic A, Mehrara BJ. Free flap reexploration: indications, treatment, and outcomes in 1193 free flaps. Plast Reconstr Surg 2007;119: 2092-100. [Crossref]

23. Esclamado RM, Carroll WR. The pathogenesis of vascular thrombosis and its impact in microvascular surgery. Head Neck 1999; 21: 355-62. [Crossref]

24. Davies DM. A world survey of anticoagulation practice in clinical microvascular surgery. Br J Plast Surg 1982; 35: 96-9. [Crossref]
25. Khouri RK. Avoiding free flap failure. Clin Plast Surg 1992; 19: 773-81. [Crossref]

26. Johnson PC, Barker JH. Thrombosis and antithrombotic therapy in microvascular surgery. Clin Plast Surg 1992; 19: 799-807. [Crossref]

27. Pugh CM, Dennis RH 2nd, Massac EA. Evaluation of intraoperative anticoagulants in microvascular free- flap surgery. J Nat Med Assoc 1996; 88: 655-7. [Crossref]

28. Ritter EF, Cronan JC, Rudner AM, Serafin D, Klitzman B. Improved microsurgical anastomotic patency with low molecular weight heparin. J Reconstr Microsurg 1998; 14: 331-6. [Crossref]

29. Collaborative overview of randomised trials of antiplatelet therapy--II: Maintenance of vascular graft or arterial patency by antiplatelet therapy. Antiplatelet Trialists' Collaboration. BMJ 1994; 308: 159-68. [Crossref]

30. Corbitt C, Skoracki RJ, Yu P, Hanasono MM. Free flap failure in head and neck reconstruction. Head Neck 2014; 36: 1440-5. [Crossref] 